\title{
MITTEILUNG
}

\section{Nachwuchspreise der Deutschen Vereinigung für Politische Wissenschaft (DVPW)}

Vorstand und Beirat der DVPW haben die jährliche Vergabe von zwei mit jeweils 1.000,- EURO dotierten Nachwuchspreisen ab dem Jahre 2002 beschlossen. Ein Preis wird für eine Dissertation vergeben, ein weiterer für eine Monographie nach der Promotion.

Folgende Bedingungen gelten:

1. In Frage kommende Publikationen für beide Preise sollen im Vorjahr des Vergabejahres veröffentlicht worden sein.

2. Die potenziellen Nachwuchspreisträger bzw. -trägerinnen dürfen nicht zur Gruppe der Hochschullehrer und -lehrerinnen bzw. Gleichgestellter gehören.

3. Für die beiden Nachwuchspreise sind Selbstbewerbungen möglich, aber selbstverständlich sind Vorschläge von Dritten willkommen.

4. Frauen sind besonders aufgefordert, sich zu bewerben; dies gilt auch für die Benennung von Kandidatinnen durch Dritte.

5. Die Einsendefrist für im Jahre 2001 veröffentlichte Publikationen ist der 31. Oktober 2002; für im Jahre 2002 veröffentlichte Publikationen wurde als Einreichungsfrist der 30. März 2003 festgelegt.

Vorschläge und Benennungen für die Wissenschaftspreise bitten wir Sie, zusammen mit einem Exemplar der Veröffentlichung an die Geschäftsstelle der DVPW zu senden. Vorstand und Beirat werden die Vorschläge durch eine aus drei Kolleginnen und Kollegen bestehende Jury begutachten lassen.

Deutsche Vereinigung für Politische Wissenschaft, c/o Universität Osnabrück, FB 1 Sozialwissenschaften, D-49069 Osnabrück, www.dvpw.de 